\section{Nicht-staatliche Akteure stärken!}

\author{
Das Problem internationaler (Umwelt-) Externalitäten wird immer wichtiger in \\ einer interdependenten Welt, die immer noch aus vielen voneinander getrennten \\ wirtschaftlichen, politischen und rechtlichen Systemen besteht. Ein Lösungsan- \\ satz könnte in einem geeigneten Ausbau supranationalen Rechts bestehen. Dies \\ wird am Problem der grenzüberschreitenden Flußläufe verdeutlicht.
}

\section{Von Rainer Durth}

$\mathrm{N}$ ormalerweise geschieht die Lösung von grenzüberschreitenden Umweltproblemen im Rahmen des Völkerrechtes, demzufolge die nationalen Regierungen die einzigen legitimen Vertreter von nationalen Interessen sind. Nach den völkerrechtlichen Regeln sind zwar insbesondere ,,erhebliche und unübliche" grenzüberschreitende Umweltbelastungen nicht zulässig, die ökologische Effektivität der völkerrechtlichen Normen wird jedoch durch ihre unbestimmte Formulierung einerseits und ihre mangelnde Durchsetzbarkeit andererseits eingeschränkt. Gleichzeitig prägt das Völkerrecht jedoch in entscheidender Weise die polit-ökonomischen Bedingungen für eine Lösung von grenzüberschreitenden Umweltproblemen, indem es den nationalen Regierungen einen Alleinvertretungsanspruch in den Verhandlungen zugesteht und es ihnen i.d.R. außerdem ermöglicht, problemrelevante grenzïberschreitende Informationsflüsse zu kontrollieren (siehe Abbildung 1). Unter diesen Bedingungen werden die nationalen Regierungen zu Schlüsselakteuren für eine Lösung internationaler Oberlauf-Unterlauf-Probleme an Flußläufen: Gegen ihren Willen kann keine Lösung durchgesetzt werden. Die Regierungen sind jedoch nicht unbedingt am Gemeinwohl interessiert, sondern sie haben eigene Zielvorstellungen und werden einer grenzüberschreitenden Lösung nur dann zustimmen, wenn sie sich einen individuellen Vorteil davon versprechen. Bei dem Kalkuil, ob eine bestimmte Form der Zusammenarbeit vorteilhaft für sie ist oder nicht, werden sie z.B. auch die erwarteten Auswirkungen auf ihre eigenen Handlungsmöglichkeiten (oder die organisierter wirtschaftlicher Interessen) und ihr politisclies Prestige berücksichtigen. In nicht integrierten Räumen sind die Bedingungen für eine Lösung von Umweltproblemen an grenzïberschreiten- den Flüssen wenig effizient, weil sie den Regierungen eine Monopolstellung hinsichtlich der grenzüberschreitenden Verhandlungen und Informationsflüsse einräumen.

Die Bedingungen zwischen integrierten Staaten - wie in der Europäischen Union - werden dagegen neben dem Völkerrecht auch durch das Recht der jeweiligen Integrationsgemeinschaft geprägt. Außerdem führt wirtschaftliche Integration dazu, daß die Schnittstellen zwischen den nationalen Rechtskörpern sauberer definiert werden müssen. Innerhalb von Integrationsräumen ist internationales Privatrecht weiter entwickelt als zwischen nicht integrierten Staaten. Vor allem das Gemeinschaftsrecht aber fuihrt zu einem dichten und vielschichtigen grenzüberschreitenden Beziehungsgeflecht zwischen allen von dem Problem betroffenen Akteuren.

Auch wenn das europäische Umweltrecht weder vollständig noch kohärent ist und die vorhande-
Quelle: vereinfachte Darstellung nach Durth 1996 nen Kontroll- und Sanktionsmechanismen bislang eher schwach ausgeprägt sind, beeindruckt der differenzierte Ansatz innerhalb der Europäischen Union gerade im Vergleich mit den Kooperationsproblemen in nicht integrierten Räumen. Im Falle der Gewässerverschmutzung beispielsweise gibt das Gemeinschaftsrecht nicht nur Emissions- und Immissionsnormen vor, sondern es gestaltet mit sogenannten horizontalen Maßnahmen auch gezielt die Rahmenbedingungen des ,politischen Marktes“ für Umweltverschmutzungen und die Transaktionskosten bestimmter Akteure. Die Gewinner der Integration sind in erster Linie Private, die ihre Nutzungsinteressen grenzüberschreitend geltend machen wollen.

Während sich ihre Handlungsmöglichkeiten unter den Bedingungen des Völkerrechts und des internationalen Privatrechts de facto darauf beschränken, daß die Bürger ihre eigene Regierung zu internationalen Verhandlungen drängen (bzw. davon abhalten) können, besteht für private Interessengruppen innerhalb der EU die Möglichkeit zwischen vier Handlungsalternativen zu wählen (vgl. auch Abb. 2):

- Sie können sich erstens weiterhin von ihren Regierungen im Ausland vertreten lassen.

- Zweitens können sie sich gezielt Informationen aus dem Ausland beschaffen und auch selber ausländische Stellen gezielt informieren.

- Drittens können sie in direkte Verhandlungen mit den ausländischen Verschmutzern (oder ,Opfern") treten und

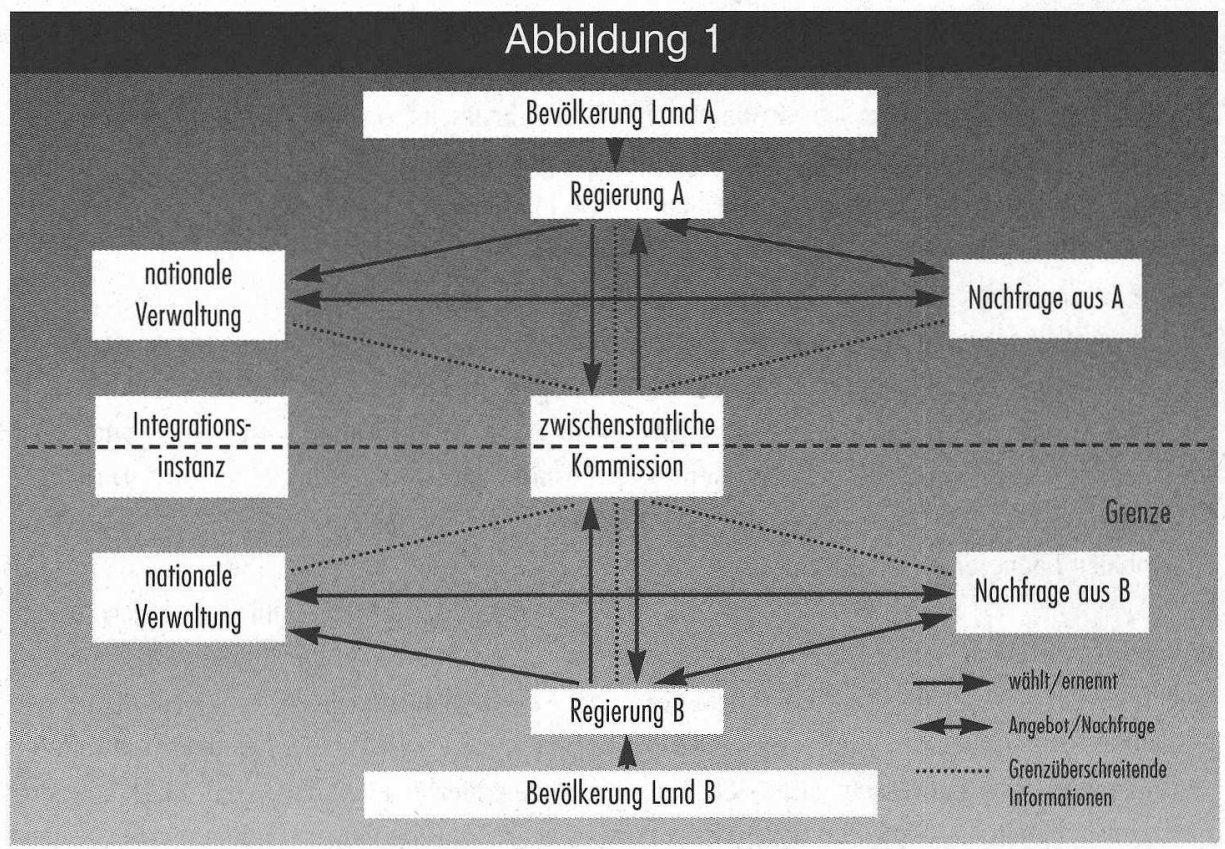




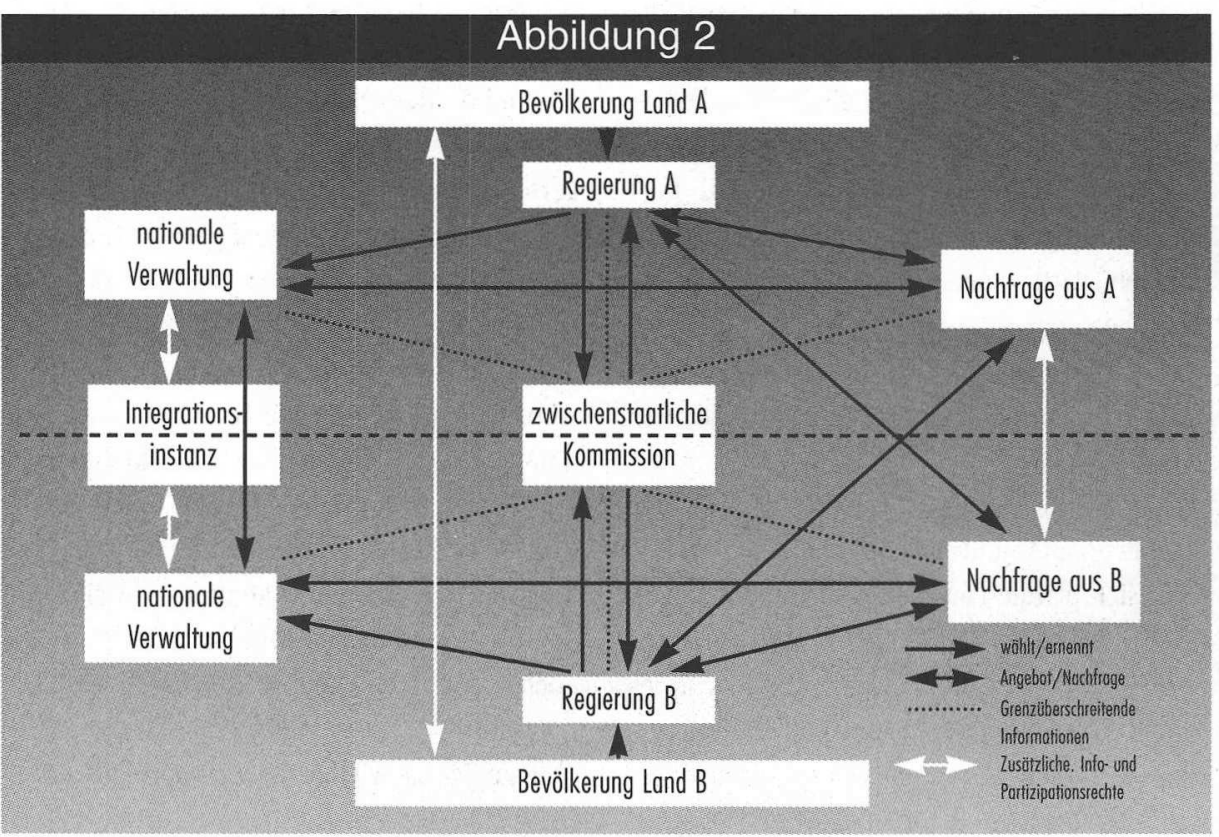

Quelle: stark vereinfachte Darstellung nach Durth 1996

- viertens dürfen sie direkt an Verwaltungsverfahren im Ausland partizipieren.

Jede Interessengruppe, die innerhalb der EU auf grenzüberschreitende Umweltbelastungen Einfluß nehmen will, steht vor der Optimierungsaufgabe, diejenige Kombination unter diesen vier Handlungsalternativen auszuwählen, die das beste Kosten-Nutzen-Verhältnis aufweist. Im Integrationsraum der EU treten Private daher als Schlüsselakteure neben die nationalen Regierungen, wenn eine Lösung für grenzüberschreitende Umweltprobleme gefunden werden soll. Die Regierungen verlieren zwar ihre Monopolstellung bezüglich der grenzüberschreitenden Verhandlungen und Informationsflüsse, aber ihre Vermittlertätigkeit bleibt auch in der EU weiterhin gefragt. Der Grund hierfür liegt in den komparativen Vorteilen, die die Regierungen hinsichtlich grenzüberschreitender Verhandlungen haben.

\section{- Rotterdamer Hafen als Beispiel}

Das systematische Vorgehen des Rotterdamer Hafens in den letzten zehn Jahren zeigt auf anschauliche Weise, daß private Interessengruppen auch tatsächlich die gesamte Bandbreite der neuen Handlungsmöglichkeiten nutzen. Und es macht deutlich, welche Bedeutung die Veränderungen des politischen Marktes innerhalb der EU für die Lösung grenzüberschreitender Umweltprobleme haben. Mitte der 80er Jahre wurde festgestellt, daß die Rotterdamer Hafensedimente hochtoxisch waren und auf einer Mülldeponie gelagert werden mußten; der größte Teil der Schadstoffe und Schwermetalle wurde in den Oberliegerstaaten freigesetzt. Die Baukosten für die Deponie betrugen 200 Mio. Gulden, und ihre Aufnahmekapazität reichte für 15 Jahre. Um zu verhindern, daß ähnliche Kosten nach 15 Jahren wieder anfallen würden, startete der Rotterdamer Hafen ein zehnjähriges Aktionsprogramm: Erstens beauftragte er ein Ingenieurbüro damit, Belastungsbilanzen für den ganzen Rhein und seine wichtigsten Nebenflüsse in den Niederlanden, Deutschland, Frankreich und der Schweiz zu erstellen. Zweitens bezahlte der Hafen ein ausländisches PR-Büro dafür, die Probleme mit den Hafensedimenten stromaufwärts bekanntzumachen und für die Sanierungsforderungen des Rotterdamer Hafens zu werben. Drittens ließ er umfangreiche juristische Expertisen erstellen, die sowohl die Ausgangsbasis für direkte Verhandlungen mit den wichtigsten Rheinverschmutzern bildeten, als auch zur Begründung von Haftungsansprüchen in grenzüberschreitenden zivilrechtlichen Prozessen dienten. Und viertens setzte der Rotterdamer Hafen durch, daß seine Forderung nach sauberen Flußsedimenten von den Regierungen der Rheinanliegerstaaten als eines von vier Kooperationszielen in das Rheinaktionsprogramm aufgenommen wurde.

Aus eigenem wirtschaftlichen Interesse wurde der Rotterdamer Hafen also zu einem Fürsprecher für einen saubereren Rhein. Aufgrund der

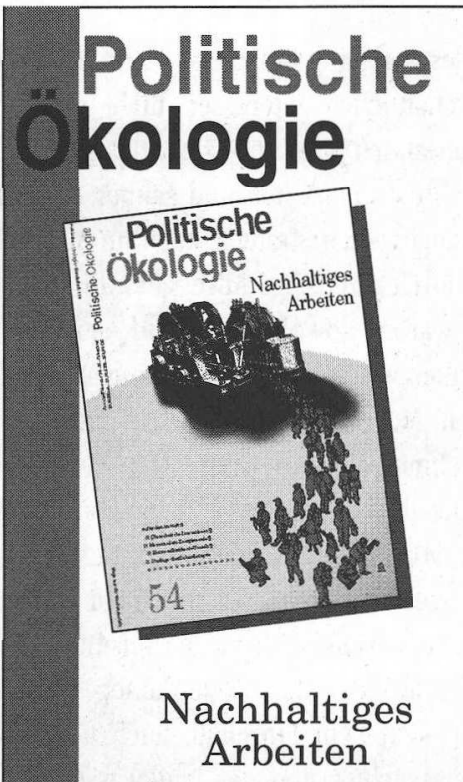

Der Arbeitsgesellschaft geht die Arbeit aus - so scheint es. Immer weniger arbeiten immer mehr. Immer mehr haben keine Erwerbsarbeit. Eine Erwerbsarbeit, die steigenden Wohlstand versprach, aber auch die Menschen wie die Natur gleichermaßen auszehrte.

Die Krise der Arbeit verlangt nach einem vollkommen neuen Umgang mit Arbeit. Aber wie könnte dieser aussehen? Wie muß eine Arbeit beschaffen sein, die sowohl die sozialen als auch die ökologischen Erfordernisse im Blick hat? Namhafte Autorinnen und Autoren diskutieren in der Politischen Ökologie die Voraussetzungen einer für Mensch und Umwelt gleichermaßen nachhaltigen Arbeit, entwerfen theoretische Konzepte und berichten über Projekte, die schon heute neue Ansätze für ein anderes Arbeiten und Leben erproben.

G Scherhorn: Vom Produktionsfaktor zur selbstbestimmten Chance $\bullet$ R. Lucas: Nachhaltigkeit als Herausforderung für eine andere Arbeit $\bullet$ A. Gorz: Jenseits der Erwerbsarbeit $\bullet$ A. Biesecker: Ein neues Konzept von Teilung und Verteilung der Arbeit $\bullet \mathbf{S}$. Schön und M. Schäfer: Szenario einer nachhaltig arbeitenden Gesellschaft - E. Redler: Über Stellenwert, Möglichkeiten und Grenzen der Eigenarbeit F. Bergmann: Mit der Neuen Arbeit neue Lebensformen erproben - U. Beck: Bürgerarbeit • G. Mutz: Tätigkeitsgesellschaft

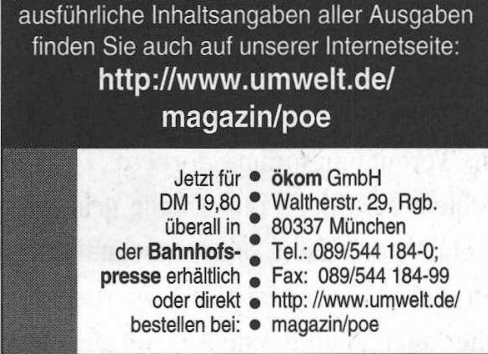


EU-Gesetzgebung konnte er seine partikulären wirtschaftlichen Interessen nicht nur in den Niederlanden äußern, sondern sie mit Hilfe einer breit angelegten und sauber durchdachten langfristigen Strategie auch im Ausland selber durchsetzen. Die Adressaten dieser Strategie waren sowohl in- und ausländische Politiker, welche auf Veränderungen der öffentlichen Meinung reagierten, als auch private Verschmutzer. Innerhalb von zehn Jahren konnte der Rotterdamer Hafen durch Androhung von Schadensersatzforderungen fast alle wichtigen Punkteinleiter am Rhein zu erheblichen Schadstoffminderungen verpflichten.

Die Aktivitäten des Rotterdamer Hafens am Rhein sind kein Einzelfall, auch holländische Gemüsezüchter und die Trinkwasserwerke im Rheineinzugsgebiet haben aus eigenem wirtschaftlichen Interesse wichtige grenzüberschreitende Umweltschutzmaßnahmen gefordert und erreicht. Nachdem eine Lösung der grenzuiberschreitenden Umweltprobleme am Rhein bis in die siebziger Jahre hinein ausschließlich der zwischenstaatlichen Zusammenarbeit vorbehalten war und sich Erfolge eher langsam einstellten, weisen die dezentralen Sanierungsbemühungen hier mittlerweile eine beachtliche Dynamik auf. Auf eine zwischenstaatliche Zusammenarbeit am Rhein kann wohl auch heute nicht verzichtet werden, die Erfahrungen dort zeigen aber, daß sich grenzüberschreitende Umweltprobleme zumindest teilweise durch Mechanismen zwischen privaten Akteuren lösen lassen.

\section{- Förderung privaten Handelns}

Die Erfahrungen im europäischen Umweltschutz machen deutlich, welches Potential private grenzuiberschreitende Initiativen für die Lösung von internationalen Externalitätenproblemen darstellen. Die obige Analyse legt die folgenden Politikempfehlungen zur Stärkung privater Akteure bei internationalen Umweltproblemen nahe:

- Bei vielen grenzüberschreitenden Umweltproblemen sind die Regierungen Monopolisten und können sich als Makler zwischen die von dem Problem betroffenen Parteien schieben und von ihnen eine politische oder ökonomische Vermittlungsprämie fordern. Das Umweltproblem wird dann nur zufällig gelöst und das Ergebnis nur zufällig volkswirtschaftlich effizient sein. Neben den nationalen Regierungen sollten daher auch andere Akteure - lokale Verwaltun- gen, möglichst unabhängige zwischenstaatliche Kommissionen und vor allem die betroffenen privaten Parteien - die Möglichkeit erhalten, über grenzüberschreitende Umweltprobleme zu verhandeln. Schon die Möglichkeit, daß auch andere Akteure sich des Problems annehmen können, wird einen positiven Einfluß auf das Verhalten der Regierungen haben. Darüber hinaus ist es wichtig, daß das Regierungsmonopol auch bei grenzüberschreitenden Informationen abgebaut wird.

- Umweltbelastungen verursachen in der Regel private und gesellschaftliche Kosten. Weil sich private Parteien nur für die Reduzierung desjenigen Anteils der Kosten einsetzen werden, den sie selber tragen müssen, ist es sinnvoll, gezielt private wirtschaftliche Interessen an einer umweltgerechten grenzüberschreitenden Lösung zu schaffen. Ein Weg ist die Etablierung von klar definierten, durchsetzbaren und möglichst auch handelbaren Verfügungsrechten.

- Bei Umweltproblemen stehen einigen wenigen Verschmutzern i.d.R. zahlreiche Umweltinteressierte gegenüber. Letzteren fällt es oft schwer, sich unter Kosten und für geringe eigene Vorteile oder gar für das öffentliche Gut 'saubere Umwelt' zusammenzuschließen und zu engagieren. Grundsätzlich sollten daher parallel zwei Wege genutzt werden, um Umweltschützern bei ihren Organisationsschwierigkeiten zu helfen:

Erstens sollte versucht werden, die entscheidenden Parameter für das Zustandekommen von kollektivem Handeln der an einer umweltgerechten Lösung interessierten Parteien (wie Gruppengröße, Stärke und Verteilung der Präferenzen) zu beeinflussen.

Zweitens sollten, wenn erforderlich, direkte und unter Umständen materielle Hilfen bei der Organisation gewährt werden. Solche Organisa-tionshilfen sind volkswirtschaftlich sinnvoll, solange der durch die Hilfe zusätzlich ermöglichte Effizienzgewinn die Kosten der Hilfe übersteigt. Als Vorbild fuir eine solche direkte Hilfe kann die von verschiedenen niederländischen Gebietskörperschaften geleistete Unterstiitzung niederländischer Gemüsebauern bei ihrem Prozeß gegen französische Kaliminen, die am Oberlauf des Rheines massiv Salz einleiteten, dienen. Der Pro$z e ß$ wurde zu einem wichtigen Präzedenffall für andere Unterlieger des Rheines.

- Die Befürworter einer Internalisierung des externen Effektes müssen ihre Interessen in der Öffentlichkeit und ggf. vor Gericht durchsetzen. Hierbei haben sie in aller Regel gegenüber den Nutznießern der Umweltverschmutzung erhebliche Nachteile. Eine Verringerung der Transaktions-, Informations- und Partizipationskosten derjenigen Gruppen, die sich für Umweltschutz engagieren, ist daher in Hinblick auf eine effi-ziente Nutzung von nationalen und grenzüberschreitenden Ressourcen wünschenswert. Gerade dieser Forderung kommen die 'horizontalen' umweltpolitischen Regelungen in der Europäischen Union entgegen, sie verbessern insbesondere die Informationslage von privaten Akteuren, die sich für den Umweltschutz einsetzen wollen.

Auch wenn nicht alle grenzïberschreitenden Umweltprobleme einen ausschließlichen Rückgriff auf private wirtschaftliche Interessen nahelegen oder gar erlauben, können private grenzüberschreitende Aktivitäten eine wichtige Ergänzung zwischenstaatlicher Verhandlungen sein. Effizienz, Effektivität und Flexibilität von Lösungsansätzen für internationale Umweltprobleme lassen sich durch eine zweckmäßige Ausgestaltung der Randbedingungen für grenzïberschreitendes privates Handeln in entscheidender Weise verbessern.

\section{Literafur}

- Durth, R.: Grenzüberschreitende Umweltprobleme und Regionale Integration. Zur Politischen Ökonomie von Umweltproblemen an grenzüberschreitenden Flüssen. Baden-Baden 1996.

- Durth, R.: Wie lassen sich grenzüberschreitende Externalitötenprobleme lösen? In: Aussenwirtschaft, 51.Jg. (1996) IV, S.539-557.

- Durth, R.: Der Rhein - Ein langer Weg zum grenzüberschreitende Umweltschutz. In: Aus Politik und Zeitgeschichte B 7/1996, S.38-47.

- Frey, B.: Internationale Politische Ökonomie. München 1985.

- Olson, M.: Die Logik kollektiven Handelns. Tübingen 1991.

- Port of Rotterdam: 10 Jahre Projekt Untersuchung Rhein. Rotterdam 1994

Der Autor
Dr. Rainer Durth ist Mitarbeiler der Kreditanstall für
Wiederaufbau und Lehrbenuftragier an der
TU Darmstadt.
Kontakt: Kreditanstall für Wiederautbau,
Palmengarienstraße $5-9,60325$ Frankfurt,
Tel: $069 / 74313607$.


(c) 20I0 Authors; licensee IÖW and oekom verlag. This is an article distributed under the terms of the Creative Commons Attribution Non-Commercial No Derivates License (http://creativecommons.org/licenses/by-nc-nd/3.o/), which permits unrestricted use, distribution, and reproduction in any medium, provided the original work is properly cited. 